\title{
Influence of global features of a Bose-Einstein condensate on
}

\section{the vortex velocity}

\author{
H. M. Cataldo and D. M. Jezek ${ }^{\mathrm{b}}$
}

Departamento de Física, Facultad de Ciencias Exactas y Naturales, Universidad de Buenos Aires, RA-1428 Buenos Aires, Argentina and Consejo Nacional de Investigaciones Científicas y Técnicas, Argentina

Received: date / Revised version: date

\begin{abstract}
We study the way in which the geometry of the trapping potential affects the vortex velocity in a Bose-Einstein condensate confined by a toroidal trap. We calculate the vortex precession velocity through a simple relationship between such a velocity and the gradient of the numerically obtained vortex energy. We observe that our results correspond very closely to the velocity calculated through time evolution simulations. However, we find that the estimates derived from available velocity field formulas present appreciable differences. To resolve such discrepancies, we further study the induced velocity field, analyzing the effect of global features of the condensate on such a field and on the precession velocity.
\end{abstract}

PACS. 03.75.Lm Tunneling, Josephson effect, Bose-Einstein condensates in periodic potentials, solitons, vortices, and topological excitations - 03.75.Kk Dynamic properties of condensates; collective and hydrodynamic excitations, superfluid flow

\section{Introduction}

Since vortices in Bose-Einstein condensates (BECs) were first produced experimentally by Matthews et al. [1], many fascinating experiments have been developed involving quantized vortices. Most of the first experiments were performed in harmonic trapping potentials, while alternative

\footnotetext{
a e-mail: cataldo@df .uba.ar

b e-mail: djezek@df .uba.ar
}

trapping potentials have been incorporated in more recent experiments. For example, quadratic-plus-quartic polynomial potentials have been used to obtain vortex lattices in fast rotating condensates $[2,3]$. The quartic term has been introduced to stabilize the system when the angular velocity exceeds the radial angular frequency of the quadratic term $[4,5]$. In recent years, there has been a revived interest in observing vortex states in more complex nonrotating 
traps, due to their relation to persistent currents. In particular, in recent experiments on toroidal traps, Ryu et al. [6] were the first to observe stable persistent flow, while Weiler et al. [7] have observed the formation of vortices.

From a theoretical point of view, the dynamics of vortices in homogeneous liquid helium systems has been widely studied [8]. Inhomogeneous superfluids, on the other hand, may be expected to display more complex vortex-dynamics phenomena, since they give rise to several new features [9]. The experimental achievement of BECs in different types of trapping potentials makes room for a variety of forms of inhomogeneous particle densities. In this context, the study of the associated vortex velocity field in an inhomogeneous medium has acquired increased interest [10]. In an axisymmetric trap, a single off-axis vortex exhibits, in addition to the ordinary circulating velocity field of a centered vortex [9], an induced velocity field [10]. In the case of a harmonic trap, such an off-axis vortex is subjected to a precession movement [11-16] related to the above induced velocity field, which, evaluated near the vortex position, defines the vortex background velocity. An explicit solution for such an induced field in two dimensions, within the Thomas-Fermi (TF) approximation, was recently published [10]. This field was derived from the sole assumption that the divergence of the particle current density vanishes. We have verified in a previous work [16] that such an estimate agrees rather well with the simulation results for a harmonic trap near the vortex position, although such results seem to be partially affected by some kind of boundary effect, disregarded in the aforementioned theoretical study. This is more evident for the field far from the vortex, since a greater correspondence than that arising from [10] was found by considering the field corresponding to an antivortex located outside the condensate, resembling an image vortex. Similar studies have been developed in a recent work by Mason and Berloff [17] in traps presenting a translational symmetry.

In the present work we investigate the effect of boundary conditions on the vortex velocity in a toroidal trap. One would expect that for the multiply-connected condensate formed in that trap, the effect of the boundary conditions should be much more dramatic than that of previously considered trapping potentials, with consequences on the velocity field all over the condensate, and on the vortex precession velocity too. Such a velocity may be calculated by way of two different approaches [9-12]. One of them involves the gradient of the vortex energy and, in the other, the velocity turns out to be proportional to the density gradient. Although the latter method appears as more widely utilized, since it requires the only knowledge of the ground state density, here we shall show that it may lead to important errors.

This work is organized as follows. In Section 2, we describe the system and, in particular, the toroidal trapping potential we have utilized. In Section 3, we analyze the form of the ground state density depending on the number of particles involved. In Section 4, we present numerical calculations of the vortex precession velocity together with different approaches to indirect predictions. In Section 5, we describe the velocity field, emphasizing the importance 
of global effects arising from boundary conditions. Finally, in Section 6, we summarize the main conclusions of our study.

\section{Toroidal trapping potential}

We consider a Bose-Einstein condensate of Rubidium atoms confined by a toroidal trap $V_{\text {trap }}$. The Gross-Pitaevskii (GP) energy density functional has the standard form [18]

$$
E[\psi]=\int\left(\frac{\hbar^{2}}{2 m}|\nabla \psi|^{2}+V_{\text {trap }}|\psi|^{2}+\frac{1}{2} g|\psi|^{4}\right) d^{3} r
$$

where $\psi$ is the condensate wave function and $m$ is the atom mass. The coupling constant $g$ is written in terms of the $s$-wave scattering length $a$ as $g=4 \pi a \hbar^{2} / m$, where $a=98.98 a_{0}$, which is the boson scattering length corresponding to ${ }^{87} \mathrm{Rb}$ and $a_{0}$ is the Bohr radius.

The variation of $E$ with respect to $\psi$, where the number of particles is fixed, yields the GP equation [18]

$$
\left(-\frac{\hbar^{2} \nabla^{2}}{2 m}+V_{\text {trap }}+g|\psi|^{2}\right) \psi=\mu \psi
$$

where $\mu$ is the chemical potential.

We use a toroidal trapping potential utilized in recent experiments $[6,7]$, which in cylindrical coordinates reads,

$$
V_{\text {trap }}(r, z)=\frac{m}{2}\left[\omega_{r}^{2} r^{2}+\omega_{z}^{2} z^{2}\right]+V_{0} \exp \left(-2 r^{2} / \lambda_{0}^{2}\right),
$$

where $\omega_{r}$ and $\omega_{z}$ denote the radial and axial frequencies, respectively. We have set $\omega_{z}>>\omega_{r}$ to suppress excitation in the $z$ direction in order to be able to use the $2 \mathrm{D}$ form of the GP equation [19]. As a function of the radial coordinate $r$, the $2 \mathrm{D}$ potential exhibits a local maximum at the center $V_{\text {trap }}(0)=V_{0}$ and an absolute minimum at

$$
r_{m}=\frac{\lambda_{0}}{\sqrt{2}} \sqrt{\ln (\alpha)}
$$

with $\alpha=4 V_{0} /\left(m \omega_{r}^{2} \lambda_{0}^{2}\right)>1$. The corresponding value of the potential is

$$
V_{\text {trap }}\left(r_{m}\right)=\frac{V_{0}}{\alpha}[\ln (\alpha)+1]
$$

which verifies $V_{\text {trap }}\left(r_{m}\right)<V_{0}$, for all $\alpha>1$.

The parameters we have used are as follows: $\omega_{r} /(2 \pi)=$ $7.8 \mathrm{~Hz}$ and $\omega_{z} /(2 \pi)=173 \mathrm{~Hz}$. For the laser beam, we have set $V_{0}=50 \hbar \omega_{r}$ and $\lambda_{0}=6 l_{r}$, where $l_{r}=\sqrt{\hbar /\left(m \omega_{r}\right)}$. Within this set of parameters the minimum of the trapping potential is located at $r_{m}=5.55 l_{r}$. Hereafter we shall use $l_{r}$ and $\omega_{r}^{-1}$ as our length and time units, respectively. The minimum value of the potential is $V_{\text {trap }}\left(r_{m}\right)=24.4$ $\hbar \omega_{r}$, which constitutes a lower bound for the chemical potential.

\section{Ground state}

We show in Figure 1 the ground state density profile $\rho_{0}=$ $\left|\psi_{0}\right|^{2}$ as a function of $x$ at $y=0$ for systems formed by two different particle numbers, namely $N_{1}=3 \times 10^{4}$ and $N_{2}=$ $10^{5}$. These densities have been obtained by numerically solving the two-dimensional GP equation, which yields the following chemical potentials: $\mu_{1}=44.5 \hbar \omega_{r}$ and $\mu_{2}=$ $67.5 \hbar \omega_{r}$ for the particle numbers $N_{1}$ and $N_{2}$, respectively.

The form of the ground state density may be easily analyzed by means of the TF approximation. In fact, one can obtain the two-dimensional density of the ground state by using the $2 \mathrm{D}$ version of (2) and neglecting the kinetic 
H. M. Cataldo, D. M. Jezek: Influence of global features of a Bose-Einstein condensate on the vortex velocity

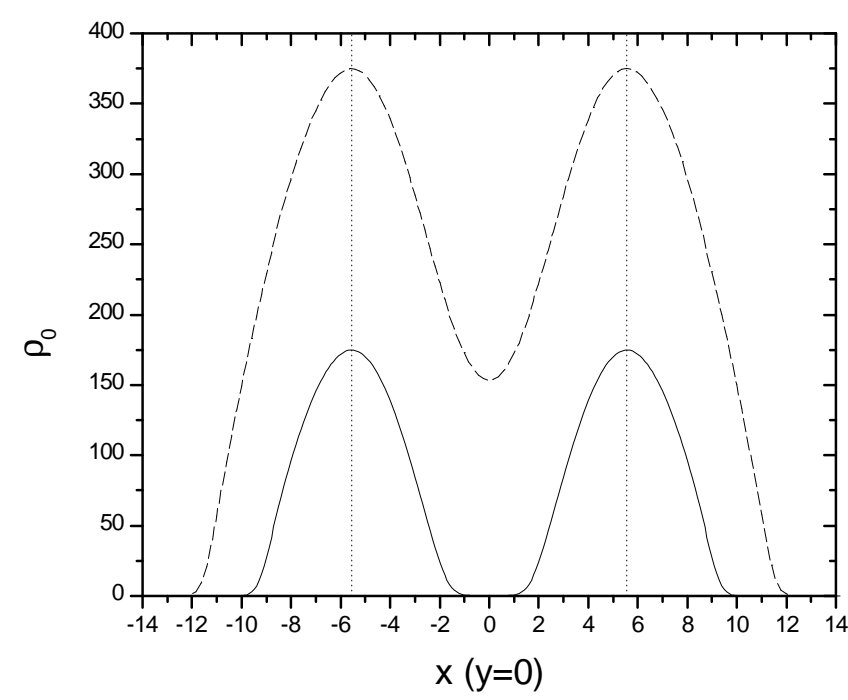

Fig. 1. Ground state density as a function of the $x$ coordinate for two different particle numbers. The full line corresponds to $N_{1}=3 \times 10^{4}$ particles, while the dashed line corresponds to $N_{2}=10^{5}$ particles. We also indicate the positions $\pm r_{m}$ of the trapping potential minimum as vertical dotted lines.

term,

$$
\left|\psi_{0}(r)\right|^{2}=\frac{1}{g_{2 D}}\left[\mu-V_{\mathrm{trap}}(r)\right] \Theta\left[\mu-V_{\mathrm{trap}}\right]
$$

where $g_{2 D}=g \sqrt{m \omega_{z} /(2 \pi \hbar)}[19]$ and $\Theta$ denotes the Heaviside function. Thus, from the above equation we can see that two types of condensates may arise depending on the value of the chemical potential. If $\mu>V_{0}$, the condensate is simply connected, while when $\mu<V_{0}$, the condensate exhibits a hole around its center. In our case, for the number of particles $N_{1}$ we have $\mu_{1}<V_{0}$ and thus the condensate presents such a hole. On the other hand, for $N_{2}$ particles, the inequality $\mu_{2}>V_{0}$ holds, and the corresponding condensate turns out to be simply connected. Finally, we can see from (6) that the potential minimum gives rise to a density maximum. In Figure 1 we have drawn vertical dotted lines at $x_{m}= \pm r_{m}$, where it may be seen that the maximum density is reached.

\section{Vortex dynamics}

In inhomogeneous media, the vortex precession velocity may be derived by way of two different approaches. One of them involves the gradient of the vortex energy and the other takes into account the background velocity field, along with effects of the vortex core. The theoretically derived precession velocity of the latter method turns out to be proportional to the density gradient. In the following Subsections we shall analyze both approaches.

\subsection{Energy gradient approach}

In inhomogeneous media, energy $E$ depends on the vortex position. In particular, for a vortex with vorticity parallel to the $z$-axis located at the point $\mathbf{r}_{0}=\left(x_{0}, y_{0}\right)$, the vortex velocity $\mathbf{v}_{p}$ may be derived from the following expression $[11,12]$

$$
2 \pi \hbar \rho_{0}\left(r_{0}\right)\left(\hat{\mathbf{z}} \times \mathbf{v}_{p}\right)=\nabla E\left(r_{0}\right)
$$

where $r_{0}=\left|\mathbf{r}_{0}\right|$. An important advantage of this approach is that, for a complex system like the present one, this energy can be calculated numerically. Thus, one can obtain the vortex velocity even when analytic expressions are difficult to derive. We have numerically computed energy $E$ as a function of the vortex position for both numbers of particles. We have found that the energy maximum is located at $r=5.3(r=5.1)$ for $N_{1}\left(N_{2}\right)$. Such a radius turns out to be slightly shifted with respect to the density 


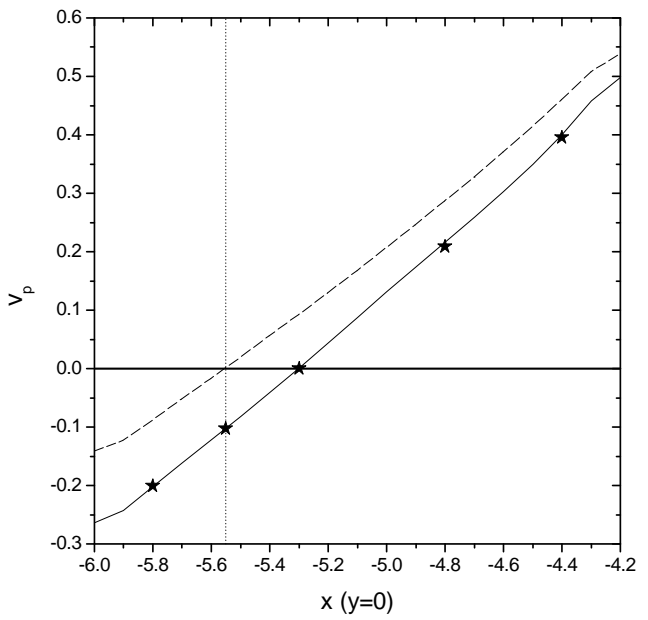

Fig. 2. Vortex precession velocity as a function of the $x$ coordinate for the condensate of $N_{1}=3 \times 10^{4}$ particles. Values derived from (7) are plotted as a full line, while the star dots indicate the temporal evolution simulations. The dashed line corresponds to the estimate given by (8). The position of the density maximum is indicated as a vertical dotted line.

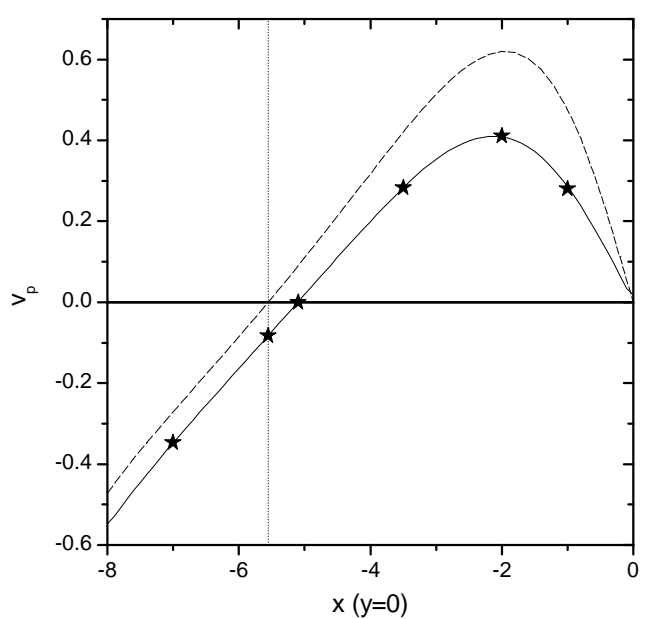

Fig. 3. Same as Figure 2 for the condensate of $N_{2}=10^{5}$ particles.

maximum. Thus, near such a critical point, the abovementioned approaches may give quite different estimates. In Figure 2 (Figure 3), we have plotted the vortex precession velocity arising from (7) for the particle number $N_{1}\left(N_{2}\right)$ as a full curve. We had to select only a small range around the density maximum in Figure 2 because the numerical calculation becomes less accurate at low densities. We have also computed the vortex velocity for several positions by means of numerical simulations of the time dependent GP equation. Such simulations were performed by means of a fifth-order Runge-Kutta algorithm. We have considered a system of size $12 \times 12(15 \times 15)$, with a grid of mesh 0.1 , for the condensate of $3 \times 10^{4}\left(10^{5}\right)$ particles. Identifying the vortex location with the position of the maximum absolute value of the velocity field, we were able to compute the time evolution of the arc length described by the vortex trajectory. The precession velocity was then calculated as the time derivative of the arc length. Small fluctuations on such a velocity $(\sim 5 \%)$ were averaged out for times much more longer than the typical time-scale of these fluctuations. Note in Figures 2 and 3 the excellent agreement between such numerical estimates (star dots) and the values predicted by formula (7). We want to stress that this approach makes no assumptions about velocity fields.

\subsection{Density gradient approach}

Much theoretical work [9-12], based on different approaches to the velocity field in inhomogeneous media, has converged at the following analytical expression for the vortex precession velocity, which involves only ground state properties:

$$
\mathbf{v}_{p}=\frac{\hbar}{2 m} \frac{\hat{\mathbf{z}} \times \nabla \rho_{0}}{\rho_{0}} \ln \left(\frac{\xi}{R}\right)
$$


where $\xi$ denotes the local value of the healing length,

$$
\xi=\sqrt{\frac{\hbar^{2}}{2^{3 / 2} m \rho_{0} g_{2 D}}},
$$

and $R$ the radial size of the condensate. For the present trapping potential, such a radius may be regarded as the external $\mathrm{TF}$ radius, arising from equating to zero expression (6). We depict in Figures 2 and 3 the precession velocity arising from equation (8), where we have used $R=9$ $(R=12)$ for the condensate of $N_{1}\left(N_{2}\right)$ particles. Note that such an expression vanishes at the density maximum, independently of the value of the condensate radius. We may see that this approximation overestimates the precession velocity. Here, it is important to notice that, in order to obtain such a theoretical estimate, no boundary conditions were taken into account. We believe that this omission may be the origin of the above-mentioned discrepancy. In particular, it is remarkable that the energy maximum, which corresponds to a stationary point, does not coincide with the density maximum. This suggests that a velocity field due to a global effect, like that induced by an image vortex which would reduce the precession velocity, may be responsible for this difference. Therefore, we think that a more careful study of the velocity field is needed. In the following Section we shall develop this idea.

\section{Velocity field}

In homogeneous media, a vortex parallel to the $z$-axis located at $\mathbf{r}_{0}$ exhibits the velocity field

$$
\mathbf{v}_{h}(\mathbf{r})=\frac{\hbar}{m} \frac{1}{\left|\mathbf{r}-\mathbf{r}_{0}\right|^{2}} \hat{\mathbf{z}} \times\left(\mathbf{r}-\mathbf{r}_{0}\right)
$$

In trapped gases, the density is no longer homogeneous and an extra velocity field is induced, which we shall call the background velocity $\mathbf{v}_{B}(\mathbf{r})$. If $\mathbf{v}(\mathbf{r})$ denotes the total field, then

$$
\mathbf{v}_{B}(\mathbf{r})=\mathbf{v}(\mathbf{r})-\mathbf{v}_{h}(\mathbf{r}) .
$$

In the following Subsections we shall distinguish between two kinds of contributions to the background velocity, one due to local inhomogeneity and the other related to global effects.

\subsection{Local effects}

Sheehy and Radzihovsky [10] have derived the following approximate expression valid in the $\mathrm{TF}$ regime near the vortex core

$$
\mathbf{v}_{B}(\mathbf{r}) \simeq \frac{\hbar}{2 m} \frac{\hat{\mathbf{z}} \times \nabla \rho_{0}\left(r_{0}\right)}{\rho_{0}\left(r_{0}\right)} \ln \left(\frac{\left|\mathbf{r}-\mathbf{r}_{0}\right|\left|\nabla \rho_{0}\left(r_{0}\right)\right|}{2 \rho_{0}\left(r_{0}\right)}\right)
$$

which was obtained through the sole assumption that $\nabla$. $\mathbf{j} \equiv 0, \mathbf{j}$ being the particle current density. Two observations may be formulated about this expression. First, the authors have disregarded any boundary condition in their calculation, second, we can see from the continuity equation that the above-mentioned assumption could only be valid outside the vortex core, where $\partial \rho_{v} / \partial t=0, \rho_{v}$ being the vortex density. Therefore, (12) may be utilized up to the border of the core at most, but not inside it, where $\partial \rho_{v} / \partial t \neq 0$. In addition, one should assume that global effects are negligible, which, as we shall see, is not the case in the present study. In Figure 4, we depict the theoretical estimate (12) together with our numerical results for 


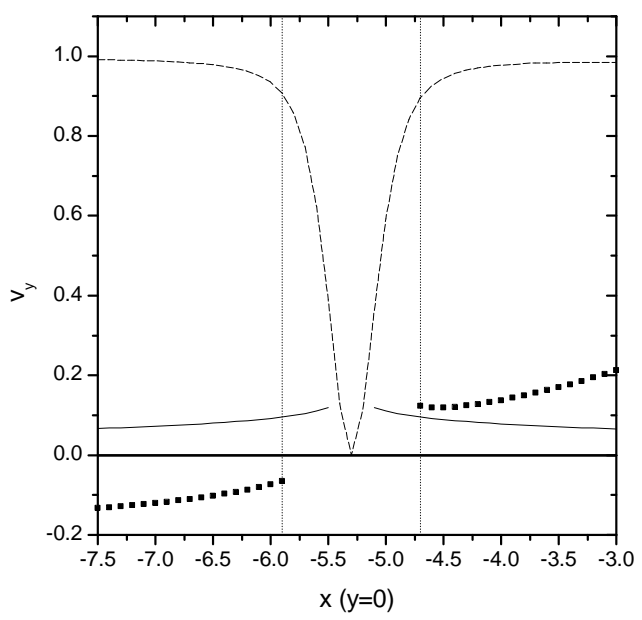

Fig. 4. The $y$-component of the background velocity as a function of the $x$ coordinate for a vortex located at $x_{0}=-5.3$ and $y_{0}=0$ in the condensate of $N_{1}=3 \times 10^{4}$ particles. The dots correspond to our numerical results, while the full line corresponds to (12). The function $f$ is depicted by a dashed line, while the core region can be approximately reduced to the zone indicated within the vertical dotted lines.

the stationary point. We have not provided any numerical estimate inside the vortex core, as the wavefunction dramatically changes in that region and the evaluation of spatial derivatives involved in the numerical calculation of the velocity field is affected by important errors. We have also plotted the function $f=\rho_{v} / \rho_{0}$. Note that $f$ is almost equal to unity outside the vortex core, which tells us that the densities with vortices $\rho_{v}$ and without vortices $\rho_{0}$ turn out to be almost the same, except in a narrow region inside the vortex core, this being a typical situation in the TF regime. Thus, the vertical lines in Figure 4 approximately separate the core region located between points where $f \simeq 0.9$. We may observe in Figure 4 that the numerically obtained velocity field exhibits a discon- tinuity around the vortex position, while the theoretical estimate does not. In deriving (12) the authors have assumed that the ground state density is a smoothly varying function around the vortex core position. To further study this issue, we have evaluated the gradient of the density $\rho_{0}$ in the neighbourhood of the border of the core. We have found that when the $x$ coordinate is varied by \pm 0.2 around $x_{0}$, the corresponding velocity arising from (12) changes about \pm 0.1 . So, the above discontinuity could be related to such a gradient, because there is no discontinuity on the numerically computed background velocity along the $y$ coordinate (cf. Figure 7), which is consistent with the symmetry of the density at both sides of the vortex core in the $y$ direction. Note that this local effect correction applies only around the vortex core, while the large disagreement encountered at larger distances seems to be related to the fact that the boundary conditions have been disregarded. We shall analyze this issue in the following Subsection.

\subsection{Global effects}

Given the above discrepancy, we believe that a revision of the validity of the assumption about the irrelevance of boundary conditions is called for. In order to avoid the contribution of local effects, we shall focus our attention on points of maximum density, which cause (12) to give a vanishing result.

In Figure 5 we plot the background velocity for a vortex located at the density maximum $\mathbf{r}_{0}=(-5.55,0)$ of the condensate with the number of particles $N_{1}$. First of 


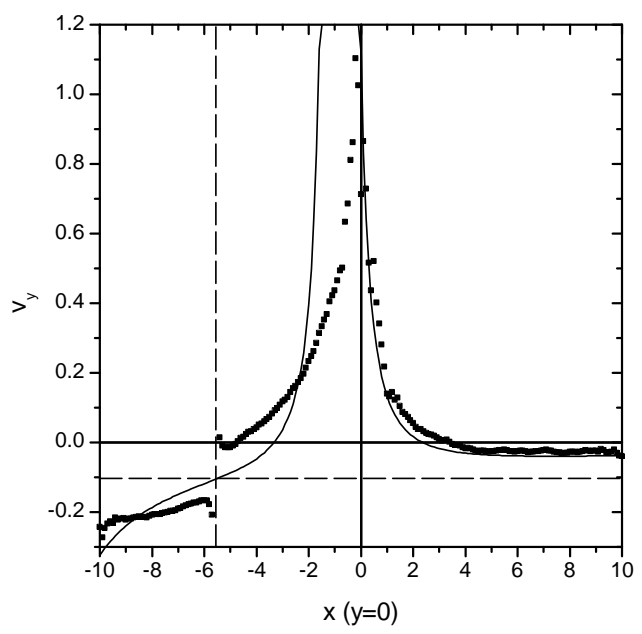

Fig. 5. The $y$-component of the background velocity as a function of the $x$ coordinate for a vortex located at $x_{0}=-5.55$ and $y_{0}=0$ in the condensate of $N_{1}=3 \times 10^{4}$ particles. The dots correspond to values arising from numerical simulations, while the full line depicts the velocity field of the vortex images, as explained in the text. Dashed horizontal and vertical lines indicate the vortex precession velocity and the vortex position, respectively.

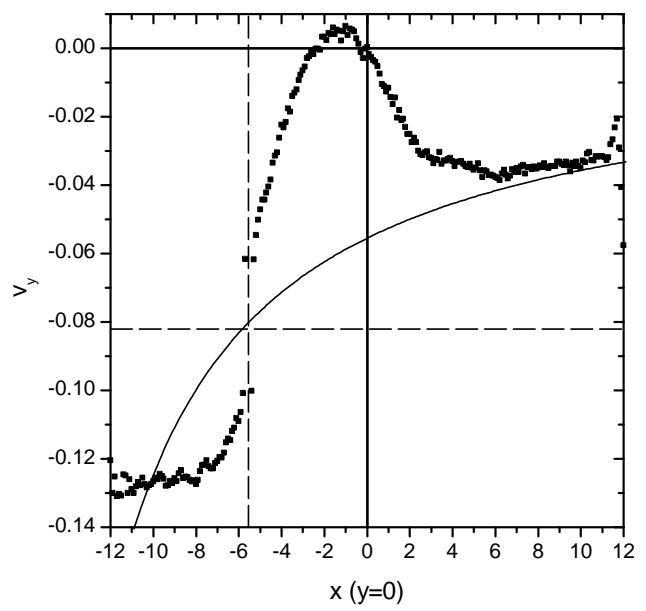

Fig. 6. Same as Figure 5 for the condensate of $N_{2}=10^{5}$ particles.

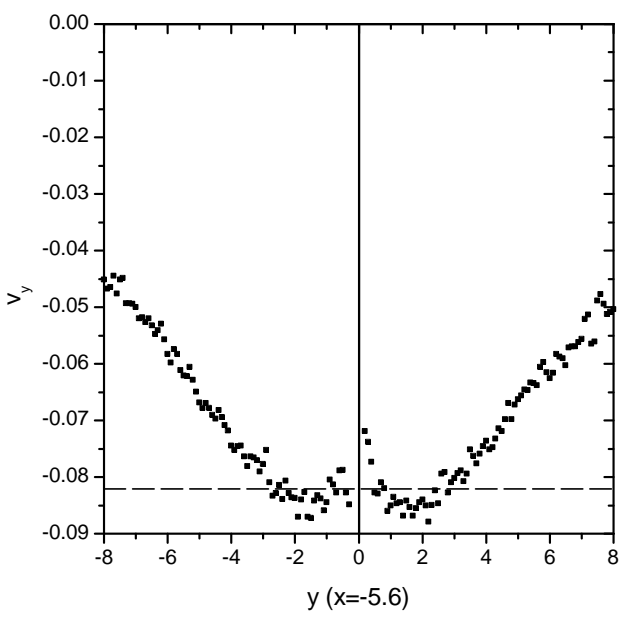

Fig. 7. The $y$-component of the background velocity as a function of the $y$ coordinate for a vortex located at $x_{0}=-5.55$ and $y_{0}=0$ in the condensate of $N_{2}=10^{5}$ particles. The dots correspond to the results arising from the numerical simulation, while the dashed horizontal line indicates the precession velocity.

all, we note that there is again a discontinuity around the vortex position. Regarding the global shape of the condensate, we would like to remind the reader that for this smaller number of particles, the ground state density exhibits a hole at its center. Thus, this condensate may be roughly approximated by a superfluid confined within two coaxial cylinders. The boundary conditions for a homogeneous fluid confined within such walls involve an infinite number of images. If the inner radius is small enough, one may consider, as a first approximation, at least three images, namely an external one and two others located within the inner cylinder, forming a dipole-like image vortex. If the radii of the internal and external cylinder walls are denoted respectively by $r_{i}$ and $R_{e}$, the field produced 
by each image is given by

$$
\mathbf{v}_{k}(\mathbf{r})=\frac{\hbar q_{k}}{m} \frac{1}{\left|\mathbf{r}-\mathbf{r}_{k}^{*}\right|^{2}} \hat{\mathbf{z}} \times\left(\mathbf{r}-\mathbf{r}_{k}^{*}\right),
$$

where their positions $\mathbf{r}_{k}^{*}$ are as follows: $\mathbf{r}_{1}^{*}=\left(-R_{e}^{2} / r_{0}, 0\right)$, $\mathbf{r}_{2}^{*}=\left(-r_{i}^{2} / r_{0}, 0\right)$, and $\mathbf{r}_{3}^{*}=\left(-\left(r_{i}^{2} / R_{e}^{2}\right) r_{0}, 0\right)$. The first image arises from the image of the vortex itself through the cylinder of radius $R_{e}$, and thus its charge is $q_{1}=-1$. The second and the third images correspond respectively to the images of the vortex and the previous image through the cylinder of radius $r_{i}$, and thus the charges are $q_{2}=-1$ and $q_{3}=1$. Therefore, this last pair may be regarded as a vortex dipole. Note that in order to preserve a fixed value of the circulation of the velocity field around a closed loop encircling the inner cylinder, one can only consider vortexantivortex pairs as image vortices inside such a cylinder.

In the case of our condensate, we may roughly estimate the contours through the values $R_{e}=8.5$ and $r_{i}=2.5$. In Figure 5, we have also plotted the velocity field of the corresponding images as a full line.

Keeping in mind that the previously reported formula (12) yields a vanishing velocity in this case, we can see that our theoretical estimate using image vortices plus the above-mentioned gap certainly provides a qualitatively good prediction. We also note from Figure 5 that the velocity field due to the images, evaluated at the vortex position, approximately coincides with the value of the precession velocity.

Finally, in Figures 6 and 7, we depict the $y$ component of the background velocity as a function of the coordinates $x$ and $y$, respectively, for a vortex located at the maximum of the ground state density of the condensate with
$N_{2}=10^{5}$ particles. In this case the condensate is simply connected, and thus the technique of using the dipole images, located near $x=0$, for describing the velocity field is no longer valid. However a 'ghost' of such a field may still be identified to the right of the vortex in Figure 6 . Although we were not able to model this effect, a single outer image vortex located at $R_{e}=10$ is shown to provide a good global estimate of the velocity, as seen in Figure 6. Moreover, this image provides, again, a good prediction of the vortex precession velocity marked as a dashed horizontal line in Figure 6. In addition, it may be seen from Figure 7 that such a precession velocity corresponds quite accurately to the background velocity around the vortex position.

\section{Concluding remarks}

We have seen that the vortex velocity value, stemming from the gradient of the numerically computed vortex energy, constitutes a very good estimate of the vortex precession velocity. We remind the reader that in this calculation, no assumption about the vortex velocity field is made. On the other hand, the expression involving the density gradient, in which a model of such a field is perfomed, is shown to overestimate the precession velocity. To resolve this discrepancy, we have investigated the influence of boundary conditions on the vortex velocity field. We have mainly focused on vortices located at the maximum of the ground state density, where previous theoretical estimates predicted a vanishing background velocity. To roughly describe such a field, we have used image 
vortices and found that several qualitative trends can be succesfully explained, particularly the lower value of the numerically computed precession velocity. To sum up, we have shown that the vortex velocity field may be affected by the condensate boundaries and that such effects can be qualitatively represented by means of image vortices.

This work has been performed under Grant PIP 5409 from CONICET.

\section{References}

1. M. R. Matthews, B. P. Anderson, P. C. Haljan, D. S. Hall, C. E. Wieman, E. A. Cornell, Phys. Rev. Lett. 83, 2498 (1999)

2. V. Bretin, S. Stock, Y. Seurin, J. Dalibard, Phys. Rev. Lett. 92, 050403 (2004)

3. S. Stock, B. Battelier, V. Bretin, Z. Hadzibabic, J. Dalibard, Laser Phys. Lett. 2 275, (2005)

4. A. L. Fetter, B. Jackson, S. Stringari, Phys. Rev. A 71, $013605(2005)$

5. J. K. Kim, A. L. Fetter, Phys. Rev. A 72, 023619 (2005)

6. C. Ryu, M. F. Andersen, P. Cladé, V. Natarajan, K. Helmerson, W. D. Phillips, Phys. Rev. Lett. 99, 260401 $(2007)$

7. C. N. Weiler, T. W. Neely, D. R. Scherer, A. S. Bradley, M. J. Davis, B. P. Anderson, Nature 455, 948 (2008)

8. R. J. Donnelly Quantized Vortices in Helium II (Cambridge University Press, Cambridge, 1991)

9. A. L. Fetter, A. A. Svidzinsky, J. Phys.: Condens. Matter 13, R135 (2001)
10. D. E. Sheehy, L. Radzihovsky, Phys. Rev. A 70, 063620 (2004)

11. E. Lundh, P. Ao, Phys. Rev. A 61, 063612 (2000)

12. S. A. McGee, M. J. Holland, Phys. Rev. A 63, 042608 (2001)

13. B. P. Anderson, P. C. Haljan, C. E. Wieman, E. A. Cornell, Phys. Rev. Lett. 85, 2857 (2000)

14. A. L. Fetter, Phys. Rev. A 69, 043617 (2004)

15. P. Mason, N. G. Berloff, A. L. Fetter, Phys. Rev. A 74, $043611(2006)$

16. D. M. Jezek, H. M. Cataldo, Phys. Rev. A 77, 043602 (2008)

17. P. Mason, N. G. Berloff, Phys. Rev. A 77, 032107 (2008)

18. E. P. Gross, Nuovo Cimento 20, 454 (1961); L. P. Pitaevskii, Sov. Phys. JETP 13, 451 (1961)

19. Y. Castin, R. Dum, Eur. Phys. J. D 7, 399 (1999) 